\title{
Erstsprachförderung bei Migrantenkindern in Kindergärten
}

\author{
Wirkungen auf phonologische Bewusstheit, Wortschatz \\ sowie Buchstabenkenntnis und erstes Lesen in der \\ Erst- und Zweitsprache
}

\author{
Urs Moser $\cdot$ Nicole Bayer $\cdot$ Verena Tunger
}

Zusammenfassung: Mit einer quasi-experimentellen Längsschnittstudie wurde bei Migrantenkindern im Kindergarten untersucht, welche Bedeutung die Förderung der Erstsprache für das Beherrschen der Erst- und Zweitsprache hat. Während zwei Jahren wurde die Sprachförderung in der Erst- und Zweitsprache mittels gleicher Themen und Geschichten im Unterricht koordiniert. Zudem wurden die Eltern dazu angeleitet, ihren Kindern zu Hause drei Mal pro Woche Geschichten in der Erstsprache vorzulesen oder Hörbücher in der Erstsprache abzuspielen.

Die aufwändige Intervention hatte weder auf die Entwicklung der Sprachkompetenzen in der Erstsprache noch auf die Entwicklung der Sprachkompetenzen in der Zweitsprache Deutsch statistisch signifikante Auswirkungen. Die aufgrund der Interdependenzhypothese von Cummins erwarteten Transfereffekte zwischen Erst- und Zweitsprache ließen sich nur sehr beschränkt nachweisen.

Schlüsselwörter: Interdependenzhypothese $\cdot$ Kindergarten · Längsschnittstudie · Migrantenkinder $\cdot$ Sprachförderung

\section{First language support for migrant children in kindergarten-The effects on phonological awareness, vocabulary, knowledge of the alphabet and reading in the first and second language}

Abstract: This contribution reports on an investigation into the importance of supporting first
language for the command of first and second language skills using a quasi-experimental longitu-

Online publiziert: 04.11 .2010

(C) VS Verlag für Sozialwissenschaften 2010

Geschäftsführer Priv.-Doz. Dr. U. Moser $(\bowtie) \cdot$ Dr. N. Bayer

Institut für Bildungsevaluation, Assoziiertes Institut der Universität Zürich,

Wilfriedstrasse 15, 8032 Zürich, Schweiz

E-Mail: urs.moser@ibe.uzh.ch

Dr. V. Tunger

Institut für Sprachwissenschaft, Universität Bern,

Länggassstrasse 49, 3000 Bern, Schweiz

E-Mail: verena.tunger@isw.unibe.ch 
dinal study of migrant children in kindergarten. For two years the development of both first and second language skills was coordinated through the treatment of the same topics and stories during lessons. Furthermore, parents were asked to read their children a story or play an audio book in the first language three times a week. This extensive intervention neither had statistically significant effects on the development of language competency in the first nor the second language, German. The transfer effects between first and second language expected by the interdependency hypothesis of Cummins were only in evidence to a very limited extent.

Keywords: Interdependency hypothesis $\cdot$ Kindergarten $\cdot$ Language development $\cdot$ Longitudinal study $\cdot$ Migrant children

\section{Einleitung}

Der Spracherwerb von Kindern mit Migrationshintergrund ist für einen erfolgreichen Lehr-Lern-Prozess in der Schule wie auch für die gesellschaftliche Integration von elementarer Bedeutung. Für die Schule ist daher die Förderung und Integration von zwei- oder mehrsprachigen Kindern zu einer wichtigen Aufgabe und zugleich zu einer besonderen Herausforderung geworden (vgl. Nohl 2007), denn im Vergleich zu monolingual aufwachsenden Kindern sind die Sprachkompetenzen von Migrantenkindern in der Zweitsprache Deutsch beim Eintritt in den Kindergarten deutlich weniger gut entwickelt, was vor allem auf Migrantenkinder aus sozioökonomisch benachteiligten Familien zutrifft (vgl. Dubowy et al. 2008; Bayer und Moser 2009).

Der bilinguale Spracherwerb führt bei Migrantenkindern häufig zu einer starken und einer schwachen Sprache, wobei beim Eintritt in den Kindergarten in der Regel die Herkunftssprache die starke Sprache ist (vgl. Kielhöfer und Jonekeit 1995; Bayer und Moser 2009). Für den Schulerfolg hingegen ist die Beherrschung der Unterrichtssprache bzw. der Bildungssprache entscheidend (vgl. Gogolin 2009). Im Hinblick auf eine erfolgreiche Schullaufbahn wird deshalb die Diskrepanz zwischen der individuell schwachen und der gesellschaftlich notwendigen starken Sprache zu einem grundlegenden Problem (Rösch 2001, S. 23 f.). Die Migrantenkinder scheitern überdurchschnittlich häufig aufgrund von ungenügenden Sprachkompetenzen in der Unterrichtssprache (vgl. Müller 1997; Baumert und Schümer 2001) sowie von oftmals eingeschränkten Unterstützungsmöglichkeiten ihres familiären Umfeldes (vgl. Maaz et al. 2007), wobei neben kulturellen und sozialen Faktoren auch noch andere Ursachen wie beispielsweise der Wohnort (vgl. Esser 2006) oder die Bildungsaspirationen der Eltern (vgl. Paulus und Blossfeld 2007) für den Bildungserfolg bedeutsam sind.

Das eigentliche Problem liegt nicht etwa darin, dass die Schule nicht auf den zunehmenden Anteil an Kindern mit Migrationshintergrund bzw. auf deren Bedürfnisse und Probleme reagiert hätte (vgl. Gomolla 2005; Ahrenholz 2008; Stamm et al. 2009). Bis heute ist es jedoch nicht gelungen, die angebotenen Stütz- und Fördermaßnahmen - insbesondere die Förderung des Zweitspracherwerbs - so zu gestalten, dass die Chancen von Migrantenkindern auf eine erfolgreiche Schullaufbahn nicht bereits beim Schuleintritt maßgeblich eingeschränkt sind (vgl. List 2005; Schründer-Lenzen und Merkens 2006; Bayer und Moser 2009). 
Die verschiedenen Programme zur sprachlichen Förderung von Kindern mit Migrationshintergrund lassen sich in fünf Kategorien einteilen (Limbird und Stanat 2006, S. 258 ff.). Im deutschen Sprachraum weit verbreitet ist der Unterricht in Deutsch als Zweitsprache ( $D a Z$ ). Dieses Modell der Sprachförderung lässt sich relativ einfach umsetzen, weil der zusätzliche Sprachunterricht vorwiegend außerhalb des Regelunterrichts stattfindet. Ebenfalls verbreitet im deutschen Sprachraum sind Modelle der strukturierten Immersion. Bei diesen Modellen wird der Unterricht den sprachlichen Voraussetzungen der Kinder angepasst. Meist sind die Lehrpersonen zweisprachig oder zumindest für den Unterricht von Deutsch als Zweitsprache qualifiziert. Häufig werden Kinder mit ungenügenden Kenntnissen der Unterrichtssprache in einer Einführungsphase auf den Regelunterricht vorbereitet. Die Integration in die Regelschule wird anschließend mit Unterricht in Deutsch als Zweitsprache unterstützt.

Weniger verbreitet im deutschen Sprachraum sind bilinguale Modelle, bei denen auch die Herkunftssprache systematisch gefördert wird. Bei bilingualen Transitions-Programmen wird zu Beginn der Schulzeit ein Teil des Unterrichts in der Herkunftssprache erteilt. Dieser Anteil wird im Laufe der Schulzeit reduziert, bis nur noch in der Zweitsprache unterrichtet wird. Bilinguale Maintenance-Programme verfolgen das Ziel, die Sprachkompetenzen in der Herkunftssprache zu erhalten. Die Sprachkompetenzen der Herkunftssprache bilden einen Teil des Curriculums. Dazu werden die Schülerinnen und Schüler nach der Herkunftssprache gruppiert. Ein Teil des Unterrichts wird während der gesamten Schulzeit in der Herkunftssprache erteilt. Bei bilingualen Two-way-Programmen werden Kinder mit zwei unterschiedlichen Erstsprachen - in der Regel eine Herkunftssprache und die Mehrheitssprache - gemeinsam unterrichtet. Idealerweise sind die beiden Gruppen gleich groß (Limbird und Stanat 2006, S. 258 ff.). Aufgrund der meist großen Anzahl an Herkunftssprachen sind bilinguale Programme wesentlich aufwändiger und schwieriger umzusetzen als monolinguale.

Welche Wirkungen monolinguale oder bilinguale Sprachprogramme insbesondere auf den Erwerb der Zweitsprache haben, ist bislang weitgehend ungeklärt (a.a. O., S. 291). Die Diskussion über das Verhältnis von Sprache und Integration sowie über die adäquate Sprachförderung von Kindern mit Migrationshintergrund wird zudem längst auch politisch geführt, wobei vor allem die Bedeutung der Erstsprache für den Erwerb der Zweitsprache kontrovers diskutiert wird (Esser 2009; Gogolin 2009).

Aus einer sprachwissenschaftlichen Perspektive sind die Hypothesen über den bilingualen Spracherwerb für die Konzeption von geeigneten Fördermaßnahmen für Migrantenkinder grundlegend. Von Bedeutung sind insbesondere Hypothesen über den Zusammenhang zwischen dem Erwerb der Erstsprache und dem Erwerb der Zweitsprache. Nach der Interdependenzhypothese von Cummins (1984) hängt die Entwicklung der Kompetenz in der Zweitsprache von den Fähigkeiten in der Erstsprache ab, weshalb beide Sprachen gleichermaßen zu fördern sind (vgl. Bialystok 2001). Die Sachlage ist jedoch alles andere als eindeutig. Verschiedene Untersuchungen weisen auf das Beherrschen der Erstsprache als notwendige Bedingung für den erfolgreichen Erwerb einer Zweitsprache hin (vgl. Baker und DeKanter 1983; Preibusch und Kröner 1987; Baur und Meder 1992; Romaine 1994; Baker und Prys-Jones 1998; Reich und Roth 2002). Es gibt aber auch Untersuchungen, die zu anderen Ergebnissen gelangen und die positive Wirkung der Kompetenzen in der Erstsprache auf die Kompetenzen in der Zweitsprache anzweifeln. 
Als entscheidend für die Sprachkompetenzen wird die Lernzeit erachtet, was als „timeon-task“-Hypothese ausgedrückt wird (vgl. Hopf 2005; Söhn 2005; Esser 2006).

Ein Blick auf einzelne Aspekte der Sprachkompetenzen, anhand derer die Bedeutung der Erstsprachkompetenzen für den Zweitspracherwerb im Sinne der Interdependenzhypothese von Cummins (1984) überprüft wurde, zeigt, dass insbesondere bei der phonologischen Bewusstheit in verschiedenen Studien ein Zusammenhang zwischen den Kompetenzen in der Erst- und der Zweitsprache nachgewiesen werden konnte. Bei anderen Aspekten der Sprachkompetenzen - beispielsweise Grammatik oder Rechtschreibung - wurden hingegen die Grenzen der gegenseitigen Beeinflussung aufgedeckt (vgl. Bialystok et al. 2005; Branum-Martin et al. 2006; Proctor et al. 2006; Wang et al. 2006).

Die Erkenntnisse über die Bedeutung der Kompetenzen in der Erstsprache für den erfolgreichen Zweitspracherwerb beruhen bisher hauptsächlich auf Studien aus dem angloamerikanischen und dem asiatischen Sprachraum. Für Europa und insbesondere für den deutschsprachigen Raum liegen keine Überblicksstudien über den Zusammenhang von doppeltem Spracherwerb bei Migrantenkindern und Schulerfolg vor. Bis anhin gibt es lediglich einige regional und thematisch enger gefasste Untersuchungen, die aufzeigen, dass sich schulische Förderung in der Erstsprache im ungünstigsten Fall neutral, in der Regel jedoch positiv auf den Zweitspracherwerb auswirkt (vgl. Reich und Roth 2002; Rosenberg et al. 2003).

Ausgehend von dieser Problemlage wurde eine Interventionsstudie konzipiert mit dem Ziel, die Wirkung einer Sprachförderung in der Erstsprache auf die Entwicklung der Sprachkompetenzen in der Erstsprache und in der Zweitsprache Deutsch zu überprüfen und somit einen Beitrag zum Zusammenhang zwischen Erst- und Zweitsprachkompetenzen zu leisten. Die Intervention wurde bei Kindern durchgeführt, die im Sommer 2006 für zwei Jahre in den Kindergarten der Stadt Zürich eintraten, und dauerte zwei Jahre. ${ }^{1}$

Mit einer Längsschnittstudie wurde untersucht, welche Bedeutung die sprachwissenschaftlich begründete Intervention für die Entwicklung der Kompetenzen in der Erstsprache und in der Zweitsprache Deutsch hat und wie die Sprachkompetenzen in der Erst- und Zweitsprache zusammenhängen. ${ }^{2}$

\section{Intervention zur Sprachförderung}

Die Intervention beruht auf der Annahme, dass eine optimale Förderung des bilingualen Spracherwerbs in der Verbindung von Maßnahmen zur Förderung der Herkunfts- und der Unterrichtssprache besteht (vgl. Cummins 1984; Reich und Roth 2002). Demgemäß wurde erwartet, dass die Abstimmung der Förderung in der Erst- und der Zweitsprache mittels gleicher Themen in den Kontexten „Vorschule“ und „Familie“ positive Auswirkungen auf den Spracherwerb hat (vgl. Codina et al. 1999).

Für die Sprachförderung im Kindergarten wurden dazu die gleichen Lerninhalte in der Erstsprachförderung und im Regelunterricht behandelt, sodass die Kinder sowohl in der Erstsprache als auch in der Unterrichtssprache Deutsch ihren Wortschatz und ihr Weltwissen erweitern konnten. Die Bedeutung der Vorschule für den Spracherwerb ist allerdings beschränkt. Der Spracherwerb ist ein Prozess, dessen Verlauf von einer Reihe interagierender Faktoren abhängt und der insbesondere durch den Zugang zur Sprache in 
sozialen Kontexten (Familie) beeinflusst wird (Ritterfeld 2000, S. 435 f.). Deshalb wurde bei der Ausgestaltung der Intervention auch berücksichtigt, dass vor allem der familiäre Zugang zur Schriftsprachkultur (Buchbesitz, Verfügbarkeit und Nutzung schriftsprachlicher Texte, Lese- und Vorlesegewohnheiten) die Sprachentwicklung in entscheidendem Ausmaß prägt (Böhme-Dürr 2000, S.447; Reich und Roth 2002, S.35; Retelsdorf und Möller 2008, S. 232 ff.).

Entsprechend den Erkenntnissen über den bilingualen Spracherwerb und über die Bedeutung sozialer Kontexte für den Schriftspracherwerb wurden für die Intervention drei Handlungsansätze bestimmt:

1. Förderung der Erstsprache im Kindergarten während zwei Lektionen pro Woche

2. Koordination der Sprachförderung in der Erstsprache und in der Zweitsprache

3. Unterstützung der Eltern bei der Förderung der Erstsprache innerhalb der Familie

Die Förderung der Erstsprache im Kindergarten wurde durch Lehrpersonen für heimatliche Sprache und Kultur ${ }^{3}$ wahrgenommen. Die Förderung der Erstsprache wurde in den Regelunterricht des Kindergartens integriert, sodass die Kinder keine zusätzlichen Stunden außerhalb des Regelunterrichts zu besuchen hatten. In den zwei zur Verfügung stehenden Lektionen zur Förderung der Erstsprache wurden jeweils 1) inhaltliche Sequenzen aus dem regulären Unterricht vertieft, 2) neue Aspekte eines Themas aufgegriffen sowie 3) kulturelle Unterschiede oder Gemeinsamkeiten bewusst gemacht.

Für die Koordination der Sprachförderung in der Erst- und Zweitsprache wurden während der Dauer von zwei Jahren vier Interventionsphasen von zwölf Wochen bestimmt. In diesen Phasen mussten während der Förderung der Erstsprache jeweils mindestens zwei Themen des Regelunterrichts aufgegriffen und in der Erstsprache vertieft werden. Zudem wurde pro Interventionsphase eine in verschiedene Sprachen übersetzte Geschichte sowohl während der Förderung der Erstsprache als auch im Regelunterricht behandelt. Ziel dieser Förderung war es, den Kindern ihr bereits vorhandenes Vokabular und ihr Weltwissen in der Erstsprache gezielt ins Gedächtnis zu rufen und damit die Kenntnisse in der Erstsprache zu festigen sowie den Erwerb der Zweitsprache Deutsch zusätzlich zu fördern.

Die Unterstützung der Eltern bei der Förderung der Erstsprache innerhalb der Familie wurde von den Lehrpersonen für heimatliche Sprache und Kultur und den Kindergartenlehrpersonen gemeinsam wahrgenommen. Die Zusammenarbeit mit den Eltern wurde durch Elternabende jeweils vor Beginn jeder der vier Interventionsphasen sichergestellt. Um eine Grundlage für eine gute Zusammenarbeit zwischen Schule und Elternhaus zu schaffen, wurden die Elternabende von den Lehrpersonen für heimatliche Sprache und Kultur geleitet. Ziel war es, die Zusammenarbeit zwischen Kindergarten und Elternhaus sorgfältig aufzubauen, damit sich die Eltern ihrer wichtigen Rolle beim Lernen ihrer Kinder bewusst wurden und diese auch wahrnahmen. Die Eltern wurden gezielt in die Sprachförderung ihrer Kinder einbezogen und aufgefordert, ihren Kindern zu Hause drei Mal pro Woche während 15 Minuten in ihrer Erstsprache vorzulesen oder in ihrer Anwesenheit Hörbücher abzuspielen. Darüber hinaus erhielten die Eltern Empfehlungen, wie sie in alltäglichen Situationen die Sprachentwicklung unterstützen können. Während der Interventionsphase fand zudem mindestens ein Treffen zwischen der Lehrperson für hei- 
matliche Sprache und Kultur und den Eltern statt, um die Bedeutung der Sprachförderung hochzuhalten und anfallende Fragen zu besprechen.

\section{Methode}

\subsection{Stichprobe}

Für die Beantwortung der Forschungsfragen wurde eine quasi-experimentelle Längsschnittstudie mit 181 Kindern der Stadt Zürich durchgeführt. Die Erstsprachen dieser Kinder waren Albanisch, Bosnisch/Kroatisch/Serbisch, Portugiesisch, Spanisch oder Tamil. Mit 63 Kindern wurde die Intervention durchgeführt (Experimentalgruppe). Der Experimentalgruppe gehörten Kinder eines Schulkreises der Stadt Zürich an, in dem schon seit mehreren Jahren Erstsprachunterricht im Kindergarten angeboten wird. Es wurden alle Kinder einbezogen, die eine der fünf Zielsprachen sprachen. Sämtliche angefragten Eltern waren bereit, aktiv am Förderprogramm teilzunehmen und die Sprachkompetenzen der Kinder regelmäßig erfassen zu lassen. Die Kontrollgruppe umfasste 118 Kinder von drei weiteren Schulkreisen der Stadt Zürich, in denen die Sprachförderung ausschließlich in der deutschen Sprache stattfindet. Die Einzugsgebiete der Schulkreise waren in Bezug auf ihre demographische Zusammensetzung vergleichbar, was anhand eines offiziellen Sozialindexes zur Belastung von Schulgemeinden kontrolliert wurde. Von 162 Kindern liegen Daten zu allen Messzeitpunkten vor.

Um die Vergleichbarkeit der beiden Gruppen zu gewährleisten, wurden verschiedene individuelle Merkmale der Kinder (Alter, Geschlecht, kognitive Grundfähigkeiten) sowie die höchste Ausbildung der Eltern im Sinne eines Indikators der sozialen Herkunft erfasst. Die kognitive Grundfähigkeit wurde mit 24 sprachfreien Aufgaben erhoben, die in Anlehnung an den Grundintelligenztest CFT 1 (vgl. Weiß et al. 1997) zu den Bereichen Ähnlichkeiten und Matrizen entwickelt wurden (Cronbach Alpha=0,81). Die soziale Herkunft und die kognitiven Grundfähigkeiten wurden zu einem Index mit Mittelwert $M=0$ und Standardabweichung $S D=1$ transformiert.

Die beiden Gruppen unterscheiden sich bezüglich der Geschlechterverteilung statistisch nicht signifikant. Der Anteil Knaben beträgt in der Experimentalgruppe 52\%, in der Kontrollgruppe $49 \%\left(\chi_{(1)}^{2}=-0,616, p=0,538\right)$. Das durchschnittliche Alter beträgt in der Experimentalgruppe 4,86 Jahre, in der Kontrollgruppe 4,94 Jahre und unterscheidet sich zwischen den Gruppen ebenfalls nicht statistisch signifikant $\left(t_{(179)}=-1,626, p=0,106\right)$. Auch in den kognitiven Grundfähigkeiten (Experimentalgruppe: $M=0,181, S D=0,939$; Kontrollgruppe: $\left.M=-0,098, S D=1,022 ; t_{(179)}=1,796, p=0,074\right)$ und in der Ausbildung der Eltern (Experimentalgruppe: $M=-0,068, S D=0,944$; Kontrollgruppe: $M=0,055$, $\left.S D=0,996 ; t_{(179)}=-0,805, p=0,422\right)$ zeigen sich zwischen den beiden Gruppen keine statistisch signifikanten Unterschiede.

\subsection{Längsschnittdesign}

Abbildung 1 zeigt das Längsschnittdesign mit den Testzeitpunkten und den Interventionsphasen. Von den Kindern der Experimental- und der Kontrollgruppe wurden während 
Abb. 1: Längsschnittdesign mit Testzeitpunkten und Interventionsphasen

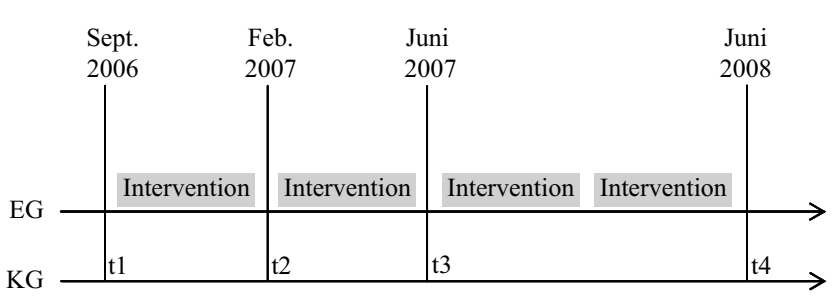

zwei Jahren insgesamt vier Mal die Sprachkompetenzen in der Erstsprache und in der Zweitsprache erfasst: kurz nach dem Eintritt in den Kindergarten, nach einem halben Jahr im Kindergarten, am Ende des ersten Jahres im Kindergarten und kurz vor dem Schuleintritt.

\subsection{Instrumente zur Erfassung der sprachlichen Kompetenzen}

Für die Erfassung der sprachlichen Kompetenzen in Deutsch wurde der Sprachteil des normierten Tests ,wortgewandt \& zahlenstark“ eingesetzt (Moser und Berweger 2007). Der Test „wortgewandt \& zahlenstark“ ist ein diagnostisches Instrument zur Erfassung sprachlicher und mathematischer Fähigkeiten von vier- bis sechsjährigen Kindern. Mit dem Sprachteil werden die phonologische Bewusstheit, der Wortschatz sowie Buchstabenkenntnis und erstes Lesen geprüft. Der Test wird als Einzelassessment durchgeführt.

Für die Erfassung der sprachlichen Kompetenzen in der Erstsprache der Migrantenkinder wurde der Sprachteil des Tests ,wortgewandt \& zahlenstark“ so weit wie möglich in die fünf Sprachen der Zielgruppe übertragen. Weil ein großer Teil der Aufgaben des Tests in deutscher Sprache die sprachlichen Fähigkeiten anhand von Lauten, Silben, Reimen oder Buchstaben überprüft, war eine Eins-zu-Eins-Übersetzung in die fünf anderen Sprachen von vornherein nicht möglich. Statt den Sprachtest zu übersetzen, wurde eine Adaptation vorgenommen. Ziel war es, äquivalente Testversionen in den genannten Zielsprachen zu erstellen, die bezüglich Umfang, Inhalt und Schwierigkeit mit dem Test in deutscher Sprache übereinstimmen. Wenn immer möglich wurden Konzept und interner Aufbau, die einer deutschen Aufgabe zugrunde liegen, beibehalten. Wenn eine Aufgabe in einer der Zielsprachen nicht funktionierte oder irrelevant war (weil beispielsweise das getestete sprachliche Phänomen nicht existiert oder peripher ist), musste eine möglichst äquivalente Aufgabe gefunden werden.

\subsection{Vergleich der Testversionen}

Bei einer Übersetzung oder Adaptation von Testinstrumenten stellt sich automatisch die Frage, ob die verschiedenen Testversionen das Gleiche messen und die einzelnen Testaufgaben gleich schwierig sind. Mit der Übersetzung bzw. Adaptation des Deutschtests in fünf Sprachversionen wurde angestrebt, dass die fünf Testversionen in der gleichen Art und Weise die Sprachkompetenzen erfassen wie die originale Version zur Erfassung der Sprachkompetenzen in Deutsch und demzufolge sozusagen von einem einheitlichen Instrument zur Erfassung der Sprachkompetenzen in sechs verschiedenen Sprachen ausgegangen werden kann. 
Damit möglichst identische Testversionen entstehen, wurde der deutschsprachige Test von Personen übersetzt, welche die Fähigkeit besitzen, die Struktur und die Ziele des originalen Tests nachzuvollziehen und die gewünschten Merkmale in die Zielsprache zu übertragen. Diese Personen mussten Kenntnisse über den Aufbau beider Sprachen besitzen und über einen sprachwissenschaftlichen Background verfügen. Damit die Ergebnisse der Sprachtests anhand verschiedener Testversionen vergleichbar sind, muss zudem gewährleistet sein, dass Kinder mit gleichen Fähigkeiten die Aufgaben unabhängig von der Testversion mit gleicher Lösungswahrscheinlichkeit beantworten können. Inwiefern die verschiedenen Testversionen gleiche Sprachkompetenzen erfassen, wurde empirisch überprüft. Dazu wurde zur Skalierung das einfache Rasch-Modell als Messmodell verwendet (vgl. Rost 2004, S. 115).

Die Modellannahmen des Rasch-Modells sind für die Entwicklung eines einheitlichen Tests zur Erfassung der Sprachkompetenzen in sechs Sprachen grundlegend. Zentral dabei sind die Itemhomogenität und die Personenhomogenität als Teil der spezifischen Objektivität (a.a.O., S.40). Zum einen müssen die Schwierigkeiten der Testaufgaben für alle Kinder gleich sein bzw. alle Testaufgaben müssen dieselbe latente Variable im Sinne einer Kompetenz ansprechen (a.a. O., S. 100). Zum andern muss für jede Person die gemessene Kompetenz unabhängig von der Auswahl der Aufgaben gemessen werden. Das bedeutet, dass alle getesteten Kinder den Test aufgrund derselben Kompetenz bearbeiten (a.a. O., S. 347 ff.).

Sofern die Aufgaben dem Rasch-Modell entsprechen, kann festgestellt werden, ob die Schwierigkeiten der Aufgaben in allen Testversionen identisch sind (Invarianz der Itemschwierigkeit). Dazu wurde die mittlere Schwierigkeit für jede Testversion auf Null zentriert. Wenn sich die Schwierigkeit von Aufgaben verschiedener Testversionen unterscheiden, dann wird vom „Differential Item Functioning“ (DIF) gesprochen (Wu et al. 2007, S.76). Ist beispielsweise eine Aufgabe in der deutschen Testversion schwierig, in der albanischen Testversion jedoch einfach, dann muss davon ausgegangen werden, dass die Aufgabe unterschiedliche Fähigkeiten misst. Für solche Unterschiede können verschiedene Gründe relevant sein. Es kann sein, dass der Sprachaufbau in den beiden Sprachen verschieden verläuft oder dass die Kinder kulturell bedingt verschieden auf die Aufgabe reagieren. Zudem können Ungereimtheiten bei der Adaptation der Ursprungsversion in die neuen Testversionen dafür ausschlaggebend sein.

Die Prüfung der Invarianz der Itemschwierigkeit in den nach Sprache differenzierten Teilstichproben erwies sich als schwierig. Der Vergleich aller sechs Testversionen (Albanisch, Bosnisch/Kroatisch/Serbisch, Deutsch, Portugiesisch, Spanisch, Tamil) führte zu einer zu geringen Anzahl an Aufgaben mit gleicher Schwierigkeit. Aus diesem Grund wurden die adaptierten Testversionen zur Erfassung der Erstsprachen jeweils separat mit der deutschen Ursprungsversion verglichen.

Die separate Passung der Testversionen auf die deutsche Ursprungsversion führte je nach Kompetenzbereich und Sprache zu einer unterschiedlich hohen Übereinstimmung bei den Aufgabenschwierigkeiten. Für den Bereich „Buchstabenkenntnis und erstes Lesen“"war es einfach, Testversionen mit gleicher Schwierigkeit zu definieren. Von den insgesamt 66 Aufgaben konnten je nach Testversionen zwischen 31 und 59 Aufgaben genutzt werden. Von den 43 Aufgaben zum Bereich „Phonologische Bewusstheit“ konnten je nach Testversion zwischen 15 und 35 Aufgaben genutzt werden. Etwas schwieriger 
war es, adäquate Testversionen für den Wortschatz zu definieren. Von den insgesamt 33 Aufgaben konnten zwischen 9 und 13 Aufgaben genutzt werden.

Weil die Anzahl Aufgaben mit gleicher Schwierigkeit je nach Testversion und Kompetenzbereich für eine valide Erfassung der Sprachkompetenzen zum Teil relativ klein war, wurden Aufgaben mit identischen Schwierigkeitsparametern als sogenannte Link-Items genutzt. Das heißt, dass zur Schätzung der Sprachkompetenzen der Schülerinnen und Schüler die Schwierigkeitsparameter der Link-Items jeweils fixiert (anchored), jene der restlichen Aufgaben jedoch variabel genutzt wurden (Wright und Stone 1979, S. 97 ff.; Kolen und Brennan 2004, S. 278). Durch die Verbindung der Testversionen anhand von Link-Items können sämtliche Aufgaben der Testversionen, die Rasch-konform sind, genutzt werden.

Für die Skalierung wurden die Schwierigkeitsparameter des Tests „wortgewandt \& zahlenstark" (Moser und Berweger 2007) importiert. Der Test wurde bei rund 1.000 Kindern der Deutschschweiz so normiert, dass der Mittelwert bei 500 Punkten und die Standardabweichung bei 100 Punkten liegen (Bayer und Moser 2009). Dies hat zur Folge, dass die Ausgangsmittelwerte der Experimental- und Kontrollgruppe in der Erst- und der Zweitsprache über oder unter 500 Punkten liegen können.

\subsection{Methoden der Auswertung}

Die Auswertung der Daten erfolgte entsprechend der hierarchischen Struktur von Längsschnittdaten im Bildungswesen (Klasse, Individuum, Testzeitpunkt) durch das „Hierarchical Linear Modelling“ (HLM) von Raudenbush und Bryk (2002). Weil die Längsschnittstudie so angelegt war, dass nicht ganze Klassen, sondern einzelne Kinder einbezogen wurden, kamen Zweiebenenmodelle zur Anwendung. Dadurch konnten die Abhängigkeit der Daten infolge des mehrmaligen Messens berücksichtigt und angemessene Standardfehler berechnet werden.

Der Lernzuwachs wurde als nicht linear angenommen, weshalb die Ergebnisse mittels quadratischer Wachstumsmodelle berechnet wurden ${ }^{4}$ (Langer 2009, S. 241 ff.). Dadurch wird neben dem linearen Effekt (Slope $\beta 1$ ) auch nachweisbar, wie der Lernfortschritt über die vier Testzeitpunkte hinweg verläuft (Slope $\beta 2$ ). Bei der Prüfung der Ausgangsmittelwerte zum Testzeitpunkt T1 (Intercepts) und der Steigungen (Slopes) zwischen der Experimental- und der Kontrollgruppe auf statistisch signifikante Unterschiede wurde jeweils die Bedeutung der Lernvoraussetzungen (allgemeine kognitive Grundfähigkeiten, Bildungsniveau der Eltern, Geschlecht und Alter) berücksichtigt.

Für die Überprüfung der Zusammenhänge zwischen der Erst- und der Zweitsprache wurden längsschnittliche Pfadmodelle nach dem Cross-Lagged Panel Design bzw. nach dem Zwei-Variablen/Zwei-Wellen-Panelmodell (Reinecke 2005, S. 74 ff.) in AMOS 7,0 spezifiziert (Arbuckle 2006). 


\section{Ergebnisse}

\subsection{Lernzuwachs in der Erstsprache}

Tabelle 1 enthält die Ergebnisse der hierarchischen Regressionsanalyse zum Lernfortschritt in der Erstsprache, aufgeteilt nach Experimentalgruppe (EG) und Kontrollgruppe $(\mathrm{KG})$ sowie nach den individuellen Lernvoraussetzungen.

Die Ausgangsmittelwerte (Intercept) der beiden Gruppen unterscheiden sich für die phonologische Bewusstheit und den Wortschatz statistisch signifikant. Mit rund 462 Punkten liegt der Mittelwert der EG im Bereich „Phonologische Bewusstheit“ rund 39 Punkte höher als derjenige der KG. Im Wortschatz erreichen die Kinder der EG einen Ausgangsmittelwert von rund 523 Punkten. Er liegt damit rund 48 Punkte höher als derjenige der KG. ${ }^{5}$ Die Ausgangsmittelwerte für Buchstabenkenntnis und erstes Lesen unterscheiden sich zwischen den beiden Gruppen statistisch nicht signifikant. Die Kinder der KG starten unwesentlich tiefer. Die individuellen Lernvoraussetzungen beeinflussen die Ausgangsmittelwerte aller drei untersuchten Bereiche statistisch nicht signifikant.

Der lineare Lernzuwachs der Kontrollgruppe (Slope $\beta 1$ ) steigt für die Bereiche „Phonologische Bewusstheit“" und ,Wortschatz“ pro Monat rund 5 Punkte weniger stark an als jener der Experimentalgruppe. Die Unterschiede sind jedoch statistisch nicht signifikant. Die negativen Werte der Schätzer für den quadratischen Lernzuwachs (Slope $\beta 2$ ) der Experimentalgruppe in den Bereichen „Phonologische Bewusstheit“ und „Wortschatz“ weisen auf einen Dämpfungseffekt hin: Mit zunehmender Zeit verlangsamt sich der Leistungszuwachs. Bei den Kindern der Kontrollgruppe ist diese Dämpfung weniger stark vorhanden als bei den Kindern der Experimentalgruppe. Allerdings bestehen auch hier keine statistisch signifikanten Unterschiede. Für den Bereich „Buchstabenkenntnis und erstes Lesen“" weisen die Kinder der Kontrollgruppe einen etwas größeren Lernfortschritt aus, der sich jedoch ebenfalls statistisch nicht signifikant vom Lernfortschritt der Experimentalgruppe unterscheidet. Gegen Ende des zweiten Kindergartenjahres zeigen sich bei den Kontrollgruppenkindern Dämpfungseffekte, während der Lernzuwachs bei den Kindern der Experimentalgruppe stärker zunimmt. Dieser Unterschied ist ebenfalls statistisch nicht signifikant.

Ein Blick auf den Einfluss der Lernvoraussetzungen für den Lernzuwachs zeigt, dass lediglich die Ausbildung der Eltern einen statistisch signifikanten Einfluss hat. Dies allerdings auch nur auf den Lernzuwachs (4,35 Punkte) und die Dämpfungseffekte $(-0,16$ Punkte) im Bereich „Phonologische Bewusstheit“.

\subsection{Lernzuwachs in der Zweitsprache Deutsch}

Tabelle 2 enthält die Ergebnisse der hierarchischen Regressionsanalyse zum Lernzuwachs in der Zweitsprache Deutsch. Sowohl der Ausgangsmittelwert (Intercept) als auch der Lernzuwachs (Slopes) unterscheiden sich für die drei Bereiche zwischen den Kindern der Experimental- und der Kontrollgruppe statistisch nicht signifikant. Im Vergleich zu den Ergebnissen zur Erstsprache liegen die Ausgangsmittelwerte und der Lernzuwachs der beiden Gruppen näher beieinander. 
Tab. 1: Phonologische Bewusstheit, Wortschatz sowie Buchstabenkenntnis und erstes Lesen in der Erstsprache: fixe und variable Effekte

\begin{tabular}{|c|c|c|c|c|c|c|}
\hline \multirow{2}{*}{$\begin{array}{l}\text { Fixe Effekte } \\
\overline{\text { Intercept } \beta 0}\end{array}$} & \multicolumn{2}{|c|}{$\begin{array}{l}\text { Phonologische Bewusst- } \\
\text { heit Erstsprache }\end{array}$} & \multicolumn{2}{|c|}{ Wortschatz Erstsprache } & \multicolumn{2}{|c|}{$\begin{array}{l}\text { Buchstabenkennt- } \\
\text { nis und erstes } \\
\text { Lesen Erstsprache }\end{array}$} \\
\hline & Koeffizient & $S E$ & Koeffizient & $S E$ & Koeffizient & $S E$ \\
\hline Experimentalgruppe & $461,66^{* * *}$ & 8,69 & $523,04 * * *$ & 11,45 & $445,70 * * *$ & 28,56 \\
\hline Kontrollgruppe & $-38,67 * * *$ & 10,97 & $-48,20 * * *$ & 12,89 & $-14,44$ & 28,36 \\
\hline Alter (in Jahren) & 37,38 & 22,83 & 3,44 & 23,26 & $-3,79$ & 72,78 \\
\hline $\begin{array}{l}\text { Geschlecht (Basis } \\
\text { Knaben) }\end{array}$ & 20,43 & 11,39 & 8,63 & 12,62 & 27,12 & 29,10 \\
\hline $\begin{array}{l}\text { Kognitive Grundfähig- } \\
\text { keiten (z-standardisiert) }\end{array}$ & 8,76 & 6,30 & 1,41 & 7,47 & 16,01 & 15,44 \\
\hline $\begin{array}{l}\text { Ausbildung der Eltern } \\
\text { (z-standardisiert) }\end{array}$ & 6,62 & 7,43 & 11,85 & 6,74 & 26,87 & 14,98 \\
\hline \multicolumn{7}{|l|}{ Slope $\beta 1$ Monate } \\
\hline Experimentalgruppe & $24,01 * * *$ & 2,57 & $12,32 * * *$ & 2,74 & 7,25 & 6,39 \\
\hline Kontrollgruppe & $-5,32$ & 2,94 & $-5,24$ & 3,15 & 4,66 & 5,89 \\
\hline Alter (in Jahren) & $-0,73$ & 5,20 & 2,53 & 5,53 & $-0,93$ & 15,05 \\
\hline $\begin{array}{l}\text { Geschlecht (Basis } \\
\text { Knaben) }\end{array}$ & $-2,71$ & 2,76 & $-1,24$ & 2,85 & $-3,15$ & 5,98 \\
\hline $\begin{array}{l}\text { Kognitive Grundfähig- } \\
\text { keiten (z-standardisiert) }\end{array}$ & 2,17 & 1,64 & 0,70 & 1,60 & 1,92 & 3,40 \\
\hline $\begin{array}{l}\text { Ausbildung der Eltern } \\
\text { (z-standardisiert) }\end{array}$ & $4,35^{*}$ & 1,64 & 1,18 & 1,31 & 1,08 & 2,87 \\
\hline \multicolumn{7}{|l|}{ Slope $\beta 2$ Monate $^{2}$} \\
\hline Experimentalgruppe & $-0,60 * * *$ & 0,13 & $-0,34^{*}$ & 0,13 & 0,24 & 0,27 \\
\hline Kontrollgruppe & 0,14 & 0,13 & 0,09 & 0,15 & $-0,40$ & 0,24 \\
\hline Alter (in Jahren) & 0,03 & 0,25 & $-0,10$ & 0,25 & 0,10 & 0,60 \\
\hline $\begin{array}{l}\text { Geschlecht (Basis } \\
\text { Knaben) }\end{array}$ & 0,19 & 0,13 & 0,10 & 0,12 & 0,21 & 0,25 \\
\hline $\begin{array}{l}\text { Kognitive Grundfähig- } \\
\text { keiten (z-standardisiert) }\end{array}$ & $-0,07$ & 0,08 & $-0,02$ & 0,07 & $-0,03$ & 0,15 \\
\hline $\begin{array}{l}\text { Ausbildung der Eltern } \\
\text { (z-standardisiert) }\end{array}$ & $-0,16^{*}$ & 0,07 & $-0,04$ & 0,05 & $-0,06$ & 0,11 \\
\hline Variable Effekte & \multicolumn{2}{|c|}{ Varianzkomponente } & \multicolumn{2}{|c|}{ Varianzkomponente } & \multicolumn{2}{|c|}{ Varianzkomponente } \\
\hline Intercept & \multicolumn{2}{|l|}{$2.679,62 * * *$} & \multicolumn{2}{|c|}{$4.668,57 * * *$} & \multicolumn{2}{|c|}{$13.540,03 * * *$} \\
\hline Slope Monate & \multicolumn{2}{|l|}{$87,24 * *$} & \multicolumn{2}{|l|}{$132,14 * * *$} & \multicolumn{2}{|l|}{$390,28 * * *$} \\
\hline Slope Monate $^{2}$ & \multicolumn{2}{|l|}{$0,17 * *$} & \multicolumn{2}{|l|}{$0,15 * * *$} & \multicolumn{2}{|l|}{$0,54 * * *$} \\
\hline Level-1 & \multicolumn{2}{|l|}{$2.777,15$} & \multicolumn{2}{|l|}{$2.075,73$} & \multicolumn{2}{|l|}{$4.276,96$} \\
\hline
\end{tabular}

${ }^{*} \mathrm{p}<0,05 ; * * \mathrm{p}<0,01 ; * * * \mathrm{p}<0,001, S E$ robuster Standard Error 
Tab. 2: Phonologische Bewusstheit, Wortschatz sowie Buchstabenkenntnis und erstes Lesen in der Zweitsprache Deutsch: fixe und variable Effekte

\begin{tabular}{|c|c|c|c|c|c|c|}
\hline \multirow{2}{*}{$\begin{array}{l}\text { Fixe Effekte } \\
\overline{\text { Intercept } \beta 0}\end{array}$} & \multicolumn{2}{|c|}{$\begin{array}{l}\text { Phonologische Bewusst- } \\
\text { heit Deutsch }\end{array}$} & \multicolumn{2}{|c|}{ Wortschatz Deutsch } & \multicolumn{2}{|c|}{$\begin{array}{l}\text { Buchstabenkenntnis unc } \\
\text { erstes Lesen Deutsch }\end{array}$} \\
\hline & Koeffizient & $S E$ & Koeffizient & $S E$ & Koeffizient & $S E$ \\
\hline Experimentalgruppe & $391,86^{* * *}$ & 13,29 & $328,09 * * *$ & 12,74 & $420,50 * * *$ & 25,07 \\
\hline Kontrollgruppe & $-0,38$ & 15,88 & 17,48 & 15,61 & 8,40 & 28,54 \\
\hline Alter (in Jahren) & 48,09 & 27,75 & 14,32 & 23,00 & $-0,37$ & 56,38 \\
\hline $\begin{array}{l}\text { Geschlecht (Basis } \\
\text { Knaben) }\end{array}$ & $31,71^{*}$ & 15,19 & 13,39 & 13,40 & 24,20 & 35,60 \\
\hline $\begin{array}{l}\text { Kognitive Grund- } \\
\text { fähigkeiten } \\
\text { (z-standardisiert) }\end{array}$ & $47,27 * * *$ & 8,96 & $21,49 * *$ & 8,00 & 15,18 & 15,58 \\
\hline $\begin{array}{l}\text { Ausbildung der El- } \\
\text { tern (z-standardisiert) }\end{array}$ & 7,09 & 7,29 & $21,83 * * *$ & 6,39 & 9,60 & 14,80 \\
\hline \multicolumn{7}{|l|}{ Slope $\beta 1$ Monate } \\
\hline Experimentalgruppe & $17,19 * * *$ & 2,45 & $15,70 * * *$ & 2,01 & 3,22 & 5,17 \\
\hline Kontrollgruppe & 2,26 & 3,16 & $-1,87$ & 2,81 & 2,56 & 6,29 \\
\hline Alter (in Jahren) & $-2,47$ & 4,55 & 0,62 & 3,95 & 6,79 & 12,65 \\
\hline $\begin{array}{l}\text { Geschlecht (Basis } \\
\text { Knaben) }\end{array}$ & $-1,52$ & 3,13 & $-1,90$ & 2,32 & $-1,57$ & 7,46 \\
\hline $\begin{array}{l}\text { Kognitive Grund- } \\
\text { fähigkeiten } \\
\text { (z-standardisiert) }\end{array}$ & $-3,06$ & 1,78 & 0,18 & 1,28 & $-0,37$ & 3,55 \\
\hline $\begin{array}{l}\text { Ausbildung der El- } \\
\text { tern (z-standardisiert) }\end{array}$ & 2,73 & 1,44 & $-0,06$ & 1,12 & 2,55 & 2,91 \\
\hline \multicolumn{7}{|l|}{ Slope $\beta 2$ Monate $^{2}$} \\
\hline Experimentalgruppe & $-0,35 * * *$ & 0,10 & $-0,38 * * *$ & 0,09 & 0,28 & 0,21 \\
\hline Kontrollgruppe & $-0,10$ & 0,13 & 0,08 & 0,12 & $-0,22$ & 0,26 \\
\hline Alter (in Jahren) & 0,06 & 0,20 & $-0,05$ & 0,18 & $-0,26$ & 0,51 \\
\hline $\begin{array}{l}\text { Geschlecht (Basis } \\
\text { Knaben) }\end{array}$ & 0,07 & 0,14 & 0,06 & 0,11 & 0,13 & 0,30 \\
\hline $\begin{array}{l}\text { Kognitive Grund- } \\
\text { fähigkeiten } \\
\text { (z-standardisiert) }\end{array}$ & 0,13 & 0,08 & $-0,02$ & 0,06 & 0,08 & 0,15 \\
\hline $\begin{array}{l}\text { Ausbildung der El- } \\
\text { tern (z-standardisiert) }\end{array}$ & $-0,12$ & 0,07 & $-0,01$ & 0,05 & $-0,10$ & 0,12 \\
\hline Variable Effekte & \multicolumn{2}{|c|}{ Varianzkomponente } & \multicolumn{2}{|c|}{ Varianzkomponente } & \multicolumn{2}{|c|}{ Varianzkomponente } \\
\hline Intercept & \multicolumn{2}{|c|}{$6.452,74 * * *$} & \multicolumn{2}{|c|}{$5.048,31 * * *$} & \multicolumn{2}{|c|}{$10.982,66^{* * *}$} \\
\hline Slope Monate & \multicolumn{2}{|l|}{$138,93 * * *$} & \multicolumn{2}{|l|}{$22,15^{*}$} & \multicolumn{2}{|l|}{$327,34 * * *$} \\
\hline Slope Monate $^{2}$ & \multicolumn{2}{|l|}{$0,21 * * *$} & \multicolumn{2}{|l|}{0,03} & \multicolumn{2}{|l|}{$0,44^{*}$} \\
\hline Level-1 & \multicolumn{2}{|l|}{$2.005,51$} & \multicolumn{2}{|l|}{$1.489,31$} & \multicolumn{2}{|l|}{$6.179,19$} \\
\hline
\end{tabular}

${ }^{*} \mathrm{p}<0,05 ;{ }^{* *} \mathrm{p}<0,01 ; * * * \mathrm{p}<0,001, S E$ robuster Standard Error 
Die individuellen Lernvoraussetzungen beeinflussen zum Teil die Ausgangsmittelwerte, jedoch nicht den Lernzuwachs. Das Geschlecht und die kognitiven Grundfähigkeiten haben einen statistisch signifikanten Einfluss auf den Ausgangsmittelwert der phonologischen Bewusstheit. ${ }^{6}$ Die Mädchen weisen gegenüber den Knaben einen Vorsprung von rund 32 Punkten aus. Ein um eine Standardabweichung höherer Index der kognitiven Grundfähigkeiten bedeutet einen rund 47 Punkte höheren Anfangsmittelwert. Beim Wortschatz beeinflussen ebenfalls die kognitiven Grundfähigkeiten (rund 21 Punkte mehr pro Standardabweichung) sowie die Ausbildung der Eltern (rund 22 Punkte mehr pro Standardabweichung) den Mittelwert zum ersten Testzeitpunkt statistisch signifikant.

\subsection{Zusammenhang zwischen Erst- und Zweitsprache}

Abbildung 2 zeigt das Pfadmodell zur Überprüfung der kausalen Effekte zwischen Erst- und Zweitsprache. Das Modell weist für die beiden Kompetenzen „Phonologische Bewusstheit“ (PB) und „Buchstabenkenntnis und erstes Lesen“ (BL) gute Anpassungen aus (PB: $\chi^{2}=16,953, d f=10, p=0,075, C F I=0,990, R M S E A=0,072 ; \mathrm{BL}: \chi^{2}=14,144$, $d f=12, p=0,292, C F I=0,996, R M S E A=0,043)$.

Die Ergebnisse der Cross-Lagged Panel Analyse für den Bereich „Phonologische Bewusstheit“ zeigen, dass sich die Erst- wie auch die Zweitsprachkompetenzen über die Zeit hinweg relativ stark durch die Kompetenzen zum vorhergehenden Messzeitpunkt erklären lassen (PB Erstsprache: $p_{T 172}=0,59, p_{T 2 T 3}=0,71, p_{T 3 T 4}=0,60$; PB Zweitsprache: $\left.p_{T 1 T 2}=0,65, p_{T 2 T 3}=0,67, p_{T 3 T 4}=0,70\right)$. Die Abhängigkeiten bleiben über die Zeit hinweg stabil. Die angenommene enge Beziehung zwischen den beiden Sprachkompetenzen im Bereich „Phonologische Bewusstheit“ wird hingegen nicht belegt. Die kreuzverzögerten Pfadkoeffizienten deuten auf eine geringe Bedeutung der Kompetenzen in der einen Sprache für die Kompetenzen in der anderen Sprache zu einem späteren Zeitpunkt hin (PB Erstsprache auf Zweitsprache: $p_{T 1 T 2}=0,15, p_{T 2 T 3}=0,15, p_{T 3 T 4}=0,02(\mathrm{~ns})$; PB Zweitsprache auf Erstsprache: $p_{T 172}=0,17, p_{T 2 T 3}=0,12, p_{T 3 T 4}=0,09(\mathrm{~ns})$ ).

Die Ergebnisse der Cross-Lagged Panel Analyse für den Bereich „Buchstabenkenntnis und erstes Lesen“ zeigen, dass sich die Zweitsprachkompetenzen Deutsch weniger stark durch die Kompetenzen zum vorangehenden Messzeitpunkt erklären lassen als die Erstsprachkompetenzen (BL Erstsprache: $p_{T 1 T 2}=0,46, p_{T 2 T 3}=0,70, p_{T 3 T 4}=0,64$; BL Zweitsprache: $\left.p_{T 1 T 2}=0,26, p_{T 2 T 3}=0,44, p_{T 3 T 4}=0,45\right)$. Die Abhängigkeiten bleiben ab dem zweiten

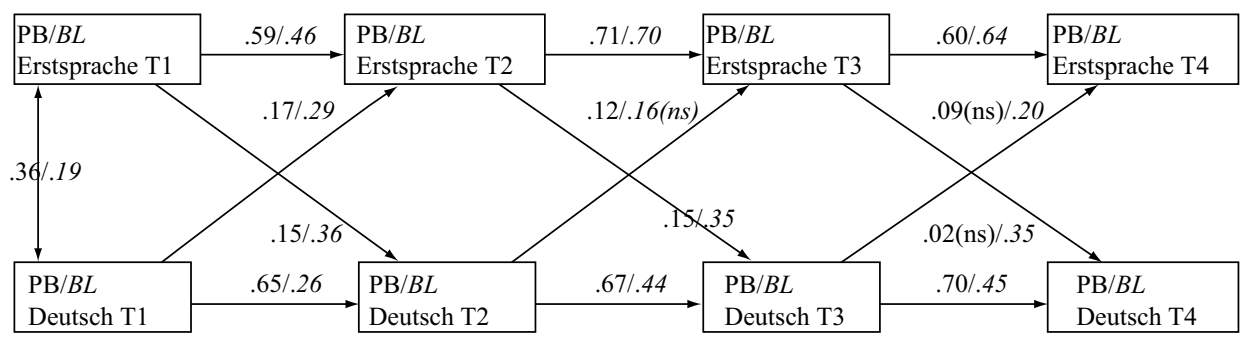

Abb. 2: Pfadmodell zum Zusammenhang zwischen Erst- und Zweitsprachkompetenzen in den Bereichen „phonologische Bewusstheit“ $(P B)$ sowie „Buchstabenkenntnis und erstes Lesen“ $(B L)$ : standardisierte Koeffizienten (ohne Kovarianz zwischen den Residuen) 
Testzeitpunkt relativ stabil. Die kreuzverzögerten Pfadkoeffizienten weisen auf Einflüsse der Kompetenzen in der einen Sprache auf die Kompetenzen in der anderen Sprache hin. Die Effekte der Kompetenzen in der Erstsprache auf die Kompetenzen in der Zweitsprache sind von geringer bis mittlerer Größe (BL Erstsprache auf Zweitsprache: $p_{T 1 T 2}=0,36$, $\left.p_{T 2 T 3}=0,35, p_{T 3 T 4}=0,35\right)$. Die Effekte der Kompetenzen in der Zweitsprache auf die Kompetenzen in der Erstsprache sind gering oder statistisch nicht signifikant (BL Zweitsprache auf Erstsprache: $\left.p_{T 1 T 2}=0,29, p_{T 2 T 3}=0,16(\mathrm{~ns}), p_{T 3 T 4}=0,20\right)$.

\section{Diskussion}

Insgesamt hat die vor allem sprachwissenschaftlich begründete Intervention weder auf die Sprachkompetenzen in der Erstsprache noch auf die Sprachkompetenzen in der Zweitsprache Deutsch eine statistisch signifikante Auswirkung. Die Verbindung von Sprachfördermaßnahmen in der Erst- und Zweitsprache sowie der Einbezug der Eltern in die Sprachförderung ihrer Kinder erzielen in dieser Form keine nachweisbaren Effekte auf die Sprachkompetenzen. Die erwarteten Effekte der Erstsprachkompetenzen auf die Zweitsprachkompetenzen gemäß der Interdependenzhypothese von Cummins (1984) ließen sich nur beschränkt nachweisen. Lediglich für den Bereich „Buchstabenkenntnis und erstes Lesen“"zeigte sich ein stabiler, geringer bis mittlerer Effekt der Kompetenzen in der Erstsprache auf die Kompetenzen in der Zweitsprache.

Dass sich die erwarteten Transfereffekte trotz zweijähriger Intervention nicht nachweisen ließen, kann mehrere Gründe haben. Die Interdependenzhypothese von Cummins (1984) bezieht sich nicht spezifisch auf den bilingualen Spracherwerb von Kindern mit Migrationshintergrund, denen häufig sowohl Sprachvorbilder in der Zweitsprache innerhalb der Familie als auch Möglichkeiten des Spracherwerbs in natürlichen sozialen Kontexten fehlen. Genau darin liegt aber das Problem für Kinder mit Migrationshintergrund aus sozioökonomisch benachteiligten Familien. Die Bedingungen der sprachlichen Sozialisation sind für die meisten Kinder mit Migrationshintergrund alles andere als optimal. Der doppelte Spracherwerb an sich stellt kein Problem dar. Die Lerngelegenheiten der Kinder bzw. die Anzahl Kontaktjahre mit Deutsch als Zweitsprache und die Kontaktqualität scheinen hingegen zu gering zu sein, als dass Transfereffekte praktisch bedeutsam werden (Reich 2008). Schließlich stand für die Sprachförderung auch nicht mehr Zeit zur Verfügung als bis anhin, sondern es wurde ein didaktisches Konzept innerhalb des regulären Kindergartenunterrichts umgesetzt.

Die Intervention könnte sich folglich bezüglich der Dauer und Intensität des bilingualen Spracherwerbs als ungenügend erwiesen haben. Die einbezogenen Migrantenkinder sind bis zum Eintritt in den Kindergarten mehrheitlich monolingual aufgewachsen. Der bilinguale Sprachunterricht der Migrantenkinder setzt faktisch erst beim Eintritt in den Kindergarten ein. Allenfalls führt der bilinguale Sprachunterricht zu einem Mehrwert für beide Sprachen, wenn eine koordinierte Förderung von längerer Dauer stattfindet. Konsequent bilinguale Modelle (Immersion) oder Modelle, die eine Förderung in der Herkunftssprache anbieten und zunehmend in die Zweitsprache überleiten (Transitions-Programme), werden denn auch als weit erfolgreicher beschrieben als Modelle, bei denen die Herkunftssprache als Fach unterrichtet wird (vgl. Gogolin 2005; Reich und 
Roth 2002; Zurer Pearson 2007). Diese Erkenntnisse konnten für die Konzipierung der Intervention aus organisatorischen und finanziellen Gründen jedoch nicht genutzt werden. Auch die Mitarbeit der Eltern könnte sich als nicht ausreichend und zu unverbindlich erwiesen haben. Dem gilt es entgegenzuhalten, dass eine abschließende Befragung der Kindergartenlehrpersonen gezeigt hatte, dass der bilinguale Zugang zur Sprachförderung von den beteiligten Eltern als Wertschätzung aufgefasst wurde, was der Zusammenarbeit zwischen Schule und Elternhaus zugute kam.

Immersion ist nicht nur ein erfolgversprechendes Modell für die Sprachförderung, sondern auch außerhalb der Schule eine notwendige Voraussetzung für den bilingualen Spracherwerb und die Integration von Migrantenkindern. Eine sprachanregende Umgebung in der Zweitsprache lässt sich jedoch durch bildungspolitische Maßnahmen allein kaum erreichen. Die Schule sieht sich mit der Tatsache konfrontiert, dass die Segregation der Bevölkerung nach bildungsrelevanten und ethnischen Merkmalen vor allem in Städten so weit fortgeschritten ist, dass der natürliche Kontext für einen bilingualen Spracherwerb bzw. für den Erwerb der Zweitsprache Deutsch für viele Migrantenkinder äußerst ungünstig ist. Die ethnischen Konzentrationen in Wohngebieten erschweren den Erwerb der Landessprache maßgeblich (Esser 2006, S. 42 ff.). Diese ungünstigen Konstellationen bleiben in der Schule erhalten, weil sich die ethnolinguistische bzw. soziale Zusammensetzung der Schule kaum von jener des Wohngebiets unterscheidet.

Mit der durchgeführten Intervention lassen sich die ungenügenden Sprachkompetenzen in der Zweitsprache Deutsch der Migrantenkinder nicht beheben. Dazu scheinen langfristiger angelegte Maßnahmen und solche, die verstärkt die Eltern bzw. Familien der Kinder einbeziehen sowie ihre sprachliche Sozialisation im frühen Kindesalter betreffen, nötig.

\section{Anmerkungen}

1 In der Schweiz besucht die Mehrheit der Kinder den Kindergarten während zwei Jahren.

2 Die Längsschnittstudie wurde vom Schweizerischen Nationalfonds unterstützt.

3 Es handelt sich dabei um Lehrpersonen aus den Herkunftsländern der Kinder. Der Unterricht findet ausschließlich in der Erstsprache statt und hat zum Ziel, Sprache und Kultur des Herkunftslandes zu vermitteln.

4 Da vier Messzeitpunkte in die Berechnung einfließen, sind grundsätzlich lineare, quadratische oder kubische Modelle möglich. Die Überprüfung der drei Modelle ergab jedoch, dass die quadratischen Modelle den Daten am besten entsprechen.

5 Die unterschiedlichen Anfangsmittelwerte könnten eine Folge davon sein, dass die Stichproben nicht zufällig ausgewählt werden konnten. Weil die Unterschiede zwischen der EG und der KG nur in der Erstsprache statistisch signifikant sind, wird allerdings vermutet, dass es sich um einen Effekt der Wertschätzung der Erstsprache in den Kindergärten der EG handelt, in denen die Förderung der Erstsprache von Anfang an - also bereits zwei Monate vor dem ersten Testzeitpunkt - zum pädagogischen Konzept gehört.

6 Dass die kognitiven Grundfähigkeiten statistisch signifikant mit den Sprachkompetenzen in der Zweitsprache Deutsch, jedoch nicht mit jenen in der Erstsprache zusammenhängen, könnte daran liegen, dass die kognitiven Grundfähigkeiten auf Deutsch erfasst wurden. 


\section{Literatur}

Ahrenholz, B. (2008). Einleitung. In B. Ahrenholz (Hrsg.), Deutsch als Zweitsprache. Voraussetzungen und Konzepte für die Förderung von Kindern und Jugendlichen mit Migrationshintergrund (S. 7-16). Freiburg im Breisgau: Fillibach Verlag.

Arbuckle, J. L. (2006). Amos 7.0 user's guide. Chicago: SPSS.

Baker, C., \& Jones, S. P. (1998). Encyclopedia of bilingualism and bilingual education. Clevedon: Multilingual Matters.

Baker, K. A., \& DeKanter, A. A. (1983). Bilingual education. Lexington: Lexington Books.

Baumert, J., \& Schümer, G. (2001). Familiäre Lebensverhältnisse, Bildungsbeteiligung und Kompetenzerwerb. In J. Baumert, E. Klieme, M. Neubrand, M. Prenzel, U. Schiefele, W. Schneider, P. Stanat, K.-J. Tillmann, \& M. Weiss (Hrsg.), PISA 2000. Basiskompetenzen von Schülerinnen und Schülern im internationalen Vergleich (S.323-409). Opladen: Leske + Budrich.

Baur, R. S., \& Meder, G. (1992). Zur Interdependenz von Muttersprache und Zweitsprache bei jugoslawischen Migrantenkindern. In R. S. Baur, G. Meder, \& V. Previšic (Hrsg.), Interkulturelle Erziehung und Zweisprachigkeit (S. 109-140). Baltmannsweiler: Schneider Hohengehren.

Bayer, N., \& Moser, U. (2009). Wirkungen unterschiedlicher Modelle der Schuleingangsstufe auf den Lern- und Entwicklungsstand: Erste Ergebnisse einer Längsschnittstudie. Zeitschrift für Grundschulforschung, 2(1), 20-34.

Bialystok, E. (2001). Bilingualism in development: Language, literacy, and cognition. Cambridge: Cambridge University Press.

Bialystok, E., McBride-Chang, C., \& Luk, G. (2005). Bilingualism, language proficiency, and learning to read in two writing systems. Journal of Educational Psychology, 97(4), 580-590.

Böhme-Dürr, K. (2000). Einfluss von Medien auf den Sprachlernprozess. In H. Grimm (Hrsg.), Enzyklopädie der Psychologie: Sprachentwicklung (Bd.3, S. 433-459). Göttingen: Hogrefe.

Branum-Martin, L., Mehta, P., Fletcher, J. M., Carlson, C. D., Ortiz, A., Carlo, M., et al. (2006). Bilingual phonological awareness: Multilevel construct validation among Spanisch-speaking kindergarteners in transitional bilingual education classrooms. Journal of Educational Psychology, 98(1), 170-181.

Codina, E., Westerbeek, K., \& Wit, Y. de (1999). Van eerste naar tweede taal. Een onderzoek naar de opbrengsten van het OET-programma Trias. Unpublished manuscript, Rotterdam.

Cummins, J. (1984). Bilingualism and special education: Issues in assessment and pedagogy. Clevedon: Multilingual Matters.

Dubowy, M., Ebert, S., Maurice, J. von, \& Weinert, S. (2008). Sprachlich-kognitive Kompetenzen beim Eintritt in den Kindergarten. Ein Vergleich von Kindern mit und ohne Migrationshintergrund. Zeitschrift für Entwicklungspsychologie und Pädagogische Psychologie, 40(3), 124-134.

Esser, H. (2006). Migration, Sprache und Integration. AKI-Forschungsbilanz 4. Arbeitsstelle Interkulturelle Konflikte und gesellschaftliche Integration (AKI). Berlin: Wissenschafts-Zentrum Berlin für Sozialforschung.

Esser, H. (2009). Der Streit um die Zweisprachigkeit: Was bringt die Bilingualität? In I. Gogolin \& U. Neumann (Hrsg.), Streitfall Zweisprachigkeit - The Bilingualism Controversy (S. 69-88). Wiesbaden: VS Verlag für Sozialwissenschaften.

Gogolin, I. (2005). Erziehungsziel Mehrsprachigkeit. In C. Röhner (Hrsg.), Erziehungsziel Mehrsprachigkeit. Diagnose von Sprachentwicklung und Förderung von Deutsch als Zweitsprache (S. 13-24). Weinheim: Juventa.

Gogolin, I. (2009). Streitfall Zweisprachigkeit - The bilingualism controversy: Les préludes. In I. Gogolin \& U. Neumann (Hrsg.), Streitfall Zweisprachigkeit - The Bilingualism Controversy (S. 15-30). Wiesbaden: VS Verlag für Sozialwissenschaften.

Gomolla, M. (2005). Schulentwicklung in der Einwanderungsgesellschaft. Strategien gegen institutionelle Diskriminierung in England, Deutschland und in der Schweiz. Münster: Waxmann. 
Hopf, D. (2005). Zweisprachigkeit und Schulleistung bei Migrantenkindern. Zeitschrift für Pädagogik, 51(2), 236-251.

Kielhöfer, B., \& Jonekeit, S. (1995). Zweisprachige Kindererziehung. Tübingen: Stauffenburg.

Kolen, M. J., \& Brennan, R. L. (2004). Test equating, scaling, and linking. Methods and practices (2.Aufl.). New York: Springer.

Langer, W. (2009). Mehrebenenanalyse. Eine Einführung für Forschung und Praxis (2.Aufl.). Wiesbaden: VS Verlag für Sozialwissenschaften.

List, G. (2005). Zur Anbahnung mehr- und quersprachiger Kompetenzen in vorschulischen Bildungseinrichtungen. In K. Jampert, P. Best, A. Guadatiello, D. Holler, \& A. Zehnbauer (Hrsg.), Schlüsselkompetenz Sprache (S. 29-32). Weimar: Verlag das netz.

Limbird, C., \& Stanat, P. (2006). Sprachförderung bei Schülerinnen und Schülern mit Migrationshintergrund: Ansätze und Wirksamkeit. In J. Baumert, P. Stanat, \& R. Watermann (Hrsg.), Herkunftsbedingte Disparitäten im Bildungswesen. Vertiefende Analysen im Rahmen von PISA 2000 (S. 257-307). Wiesbaden: VS Verlag für Sozialwissenschaften.

Maaz, K., Watermann, R., \& Baumert, J. (2007). Familiärer Hintergrund, Kompetenzentwicklung und Selektionsentscheidungen in gegliederten Schulsystemen im internationalen Vergleich. Eine vertiefende Analyse von PISA-Daten. Zeitschrift für Pädagogik, 53(4), 444-461.

Moser, U., \& Berweger, S. (2007). wortgewandt \& zahlenstark: Lern- und Entwicklungsstand bei 4- bis 6-Jährigen. St. Gallen: Interkantonale Lehrmittelzentrale, Lehrmittelverlage der Kantone St. Gallen und Zürich.

Müller, R. (1997). Sozialpsychologische Grundlagen des schulischen Zweitspracherwerbs bei MigrantenschülerInnen. Aarau: Sauerländer.

Nohl, A. -M. (2007). Kulturelle Vielfalt als Herausforderung für pädagogische Organisationen. Zeitschrift für Erziehungswissenschaft, 10(1), 61-74.

Paulus, W., \& Blossfeld, H. -P. (2007). Schichtspezifische Präferenzen oder sozioökonomisches Entscheidungskalkül? Zur Rolle elterlicher Bildungsaspirationen im Entscheidungsprozess beim Übergang von der Grundschule in die Sekundarstufe. Zeitschrift für Pädagogik, 53(4), 491-508.

Preibusch, W., \& Kröner, B. (1987). Deutsch-türkische Sprachenbalance bei türkischen Fünftklässlern. Deutsch lernen, 13(4), 19-29.

Proctor, C. P., August, D., Carlo, M. S., \& Snow, C. (2006). The intriguing role of Spanish language vocabulary knowledge in predicting English reading comprehension. Journal of Educational Psychology, 98(1), 159-169.

Raudenbush, S. W., \& Bryk, A. S. (2002). Hierarchical linear models. Applications and data analysis methods. Thousand Oaks: Sage.

Reich, H. H. (2008). Die Sprachaneignung von Kindern in Situationen der Zwei- und Mehrsprachigkeit. In K. Ehlich, U. Bredel, \& H. H. Reich (Hrsg.), Referenzrahmen zur altersspezifischen Sprachaneignung (S. 163-169). Berlin: Bundesministerium für Bildung und Forschung.

Reich, H. H., \& Roth, H. J. (2002). Spracherwerb zweisprachig aufwachsender Kinder und Jugendlicher. Ein Überblick über den Stand der nationalen und internationalen Forschung. Hamburg: Behörde für Bildung und Sport.

Reinecke, J. (2005). Strukturgleichungsmodelle in den Sozialwissenschaften. München: Oldenbourg.

Retelsdorf, J., \& Möller, J. (2008). Familiäre Bedingungen und individuelle Prädiktoren der Lesekompetenz von Schülerinnen und Schülern. Psychologie in Erziehung und Unterricht, 55, 227-237.

Ritterfeld, U. (2000). Welchen und wie viel Input braucht das Kind? In H. Grimm (Hrsg.), Enzyklopädie der Psychologie: Sprachentwicklung (Bd.3, S. 433-459). Göttingen: Hogrefe.

Romaine, S. (1994). Bilingualism. Oxford: Blackwell.

Rösch, H. (2001). Zweisprachige Erziehung in Berlin im Elementar- und Primarbereich. Essener Linguistische Skripte - elektronisch, 1(1), 23-44. 
Rosenberg, S., Lischer, R., Kronig, W., Nicolet, M., Bürli, A., Schmid, P., et al. (2003). Schulund Bildungslaufbahn von immigrierten , leistungsschwachen "Schülerinnen und Schülern (Schlussbericht CONVEGNO 2002. Studien \& Berichte 19A). Bern: EDK.

Rost, J. (2004). Lehrbuch Testtheorie - Testkonstruktion. Bern: Huber.

Schründer-Lenzen, A., \& Merkens, H. (2006). Differenzen schriftsprachlicher Kompetenzentwicklung bei Kindern mit und ohne Migrationshintergrund. In A. Schründer-Lenzen (Hrsg.), Risikofaktoren kindlicher Entwicklung. Migration, Leistungsangst und Schulübergang (S. 15-44). Wiesbaden: VS Verlag für Sozialwissenschaften.

Söhn, J. (2005). Zweisprachiger Schulunterricht für Migrantenkinder (AKI-Forschungsbilanz 2). Berlin: Wissenschaftszentrum Berlin für Sozialforschung.

Stamm, M., Reinwand, V., Burger, K., Schmid, K., Viehhauser, M., \& Muheim, V. (2009). Frühkindliche Bildung in der Schweiz - ein Bericht im Auftrag der Schweizerischen UNESCOKommission. Freiburg: Universität Freiburg.

Wang, M., Park, Y., \& Lee, K. R. (2006). Korean-English biliteracy acquisition: Cross-language phonological and orthographic transfer. Journal of Educational Psychology, 98(1), 148-158.

Weiß, R. H., Cattell, R. B., \& Osterland, J. (1997). CFT 1. Grundintelligenztest Skala 1. Göttingen: Hogrefe.

Wright, B. D., \& Stone, M. H. (1979). Best test design. Rasch maesurement. Chicago: Mesa Press.

Wu, M. L., Adams, R. J., Wilson, M. R., \& Haldane, S. A. (2007). ACER ConQuest Version 2.0: Generalised item response modelling software manual. Melbourne: Australian Council for Educational Research.

Zurer Pearson, B. (2007). Social factors in childhood bilingualism in the United States. Applied Psycholinguistics, 28, 399-410. 